\title{
Superimposition of 3-dimensional cone-beam computed tomography models of growing patients
}

\author{
Lucia H. C. Cevidanes $^{a}$, Gavin Heymann ${ }^{b}$, Marie A. Cornelis ${ }^{c}$, Hugo J. DeClerck ${ }^{d}$, and J. F. \\ Camilla Tulloche \\ Chapel Hill and Durham, NC, and Brussels, Belgium
}

\section{Abstract}

Introduction-The objective of this study was to evaluate a new method for superimposition of 3dimensional (3D) models of growing subjects.

\begin{abstract}
Methods-Cone-beam computed tomography scans were taken before and after Class III malocclusion orthopedic treatment with miniplates. Three observers independently constructed 18 3D virtual surface models from cone-beam computed tomography scans of 3 patients. Separate 3D models were constructed for soft-tissue, cranial base, maxillary, and mandibular surfaces. The anterior cranial fossa was used to register the 3D models of before and after treatment (about 1 year of follow-up).
\end{abstract}

Results-Three-dimensional overlays of superimposed models and 3D color-coded displacement maps allowed visual and quantitative assessment of growth and treatment changes. The range of interobserver errors for each anatomic region was $0.4 \mathrm{~mm}$ for the zygomatic process of maxilla, chin, condyles, posterior border of the rami, and lower border of the mandible, and $0.5 \mathrm{~mm}$ for the anterior maxilla soft-tissue upper lip.

Conclusions-Our results suggest that this method is a valid and reproducible assessment of treatment outcomes for growing subjects. This technique can be used to identify maxillary and mandibular positional changes and bone remodeling relative to the anterior cranial fossa.

Among the most challenging endeavors in orthodontics is the unraveling of morphologic growth interactions between dental and skeletal components that underpin the response to treatment. ${ }^{1}$ Superimposition of lateral cephalograms has been the standard in quantification of changes from treatment and growth. ${ }^{2-10}$ Differentiating dentofacial changes caused by treatment from those induced by growth is not possible with either 2-dimensional or 3dimensional (3D) superimposition methods ${ }^{5-13}$ However, comparison of treated and untreated controls by using 3D regional superimpositions has the potential to assess bone displacements (shift in position) and remodeling (change in size and shape) of skeletal and soft-tissue facial components relative to the cranial base. This assessment might improve our interpretations of the dynamic feedback through which growth and treatment effects interact. ${ }^{11-13}$

Copyright $@ 2009$ by the American Association of Orthodontists.

Reprint requests to: Lucia H. C. Cevidanes, Department of Orthodontics, School of Dentistry, University of North Carolina, 201 Brauer Hall, CB7450, Chapel Hill, NC 27599; cevidanl@ dentistry.unc.edu..

a Assistant professor, Department of Orthodontics, School of Dentistry, University of North Carolina, Chapel Hill.

brivate practice, Durham, NC.

${ }^{c}$ Consultant, Experimental Morphology Unit and Department of Orthdontics, Université Catholique de Louvain, Brussels, Belgium.

dPrivate practice, Brussels, Belgium.

eProfessor, Department of Orthodontics, School of Dentistry, University of North Carolina, Chapel Hill.

The authors report no commercial, proprietary, or financial interest in the products or companies described in this article. 
Superimposition of cone-beam computed tomography (CBCT) scans by using registration on the whole surface of the cranial base has been validated for non-growing subjects. ${ }^{11}$ The purpose of this study was to determine the reproducibility of 3D superimpositions to evaluate overall facial changes in growing patients over time. Specifically, we assessed a method of registration on the anterior cranial fossae and the ethmoid bone, since the growth of these structures is completed in early infancy.

\section{MATERIAL AND METHODS}

The imaging protocol involved 40-second scans in a CBCT scanner (iCat, Imaging Sciences International, Hatfield, Pa) with a $16 \times 22-\mathrm{cm}$ field of view. The images were reformatted to yield a voxel size of $0.5 \mathrm{~mm}$ and then cropped to facilitate image analysis. ${ }^{12}$ Experimental protocols were approved by the Institutional Review Board of the University of North Carolina.

Three observers, 2 orthodontists and a third-year orthodontic resident, were calibrated for analysis of serial CBCT images using 2 images not included in this study. After calibration, each observer, working independently, analyzed before and after treatment CBCT scans of 3 growing patients (mean age, 11.4 years). Image analysis was done in 4 steps: model construction, image registration, transparency overlay, and quantitative measurement.

The 3 examiners created a total of 18 3D surface models using ITK-SNAP open-source software (http://www.itksnap.org). ${ }^{14}$ These surface models differ from currently available commercial software models that only display 3D projections of the face (3D rendering).

\section{Image registration}

A fully automated registration was used to mask anatomic structures displaced with growth and treatment. ${ }^{15}$ The Imagine software (http://ia.unc.edu/dev/download/imagine/index.htm) computes the rigid registration (translation and rotation) that optimally aligns before and after treatment gray level CBCT data sets with subvoxel accuracy at the anterior cranial base. ${ }^{15}$ The registration used the best anatomic fit of anterior cranial base structures that have completed growth by age 7,16-18: anterior wall of sella, anterior clinoid processes, planum sphenoidale, lesser wings of the sphenoid, superior aspect of ethmoid and cribriform plate, cortical ridges on the medial and superior surfaces of the orbital roofs, and inner cortical layer of the frontal bones (Fig 1).

The CMF software (Maurice Müller Institute, Bern, Switzerland) overlays 3D surface models that are registered in the same coordinate system (Fig 2). ${ }^{17}$ This tool shows the location and the direction of dental, bone, and soft-tissue displacements relative to the cranial base by using various transparencies that allow correlation of hard-tissue and soft-tissue changes.

The CMF software calculated surface distances and generated color maps between two 3D models (Figs 3-5). ${ }^{19}$ The color maps have about 600,000 color-coded surface distances in millimeters between before and after treatment 3D-model surface triangles, so that the difference between the 2 surfaces at any location can be quantified. Isolines (contour line tool) were used to delineate surface changes for 9 anatomic regions (Fig 3 and Table). Hard- and soft-tissue changes were described not as absolute displacements, but as displacements relative to the cranial base.

\section{RESULTS}

The changes with growth and treatment were measured on the 3D models constructed by 3 observers. Composite images of 3D superimposed color-coded maps show clearly the similarity of the surface distances along all maxillary and mandibular surfaces among the 3 
observers (Figs 3-5). The interexaminer range of measurements across anatomic regions was equal or less than $0.5 \mathrm{~mm}$ (Table).

\section{DISCUSSION}

The intent of this study was to evaluate a novel technique to describe treatment outcomes in growing patients. The choice of landmarks and structures for 3D superimposition of growing subjects has not yet been investigated.

Because 3D projections cannot be used for precise landmark location or selection of anatomic regions, this study involved creation, registration, and superimposition of 3D surface models.

${ }^{12}$ Potential sources of variations between examiners are threefold. First, low gray-scale contrast in anatomic regions with thin cortical bone prevents automatic definition of these structures' boundaries. Manual editing can introduce slightly different outlining of surface boundaries by different examiners. Second, the user input to define the properties of 3D surface model creation could also lead to minor surface variations. Third, variations in quantification of treatment outcomes could stem from examiner choice of the isoline contours on the color map. No matter what the source of between-examiner variations, the magnitude of variations in all cases was equal to or smaller than the CBCT voxel resolution. Variations of $0.5 \mathrm{~mm}$ or less are clinically insignificant.

The major strength of this method is that registration does not depend on the precision of the 3D surface models. The cranial base models are only used to mask anatomic structures that change with growth and treatment. The registration procedures actually compare voxel by voxel of gray-level CBCT images, containing only the cranial base, to calculate rotation and translation parameters between the 2 images.

Regional superimposition in the anterior cranial base does not completely define the movement of the mandible relative to the maxilla. ${ }^{5-10}$ The studies of Efstratiadis et al ${ }^{5,6}$ highlighted that cranial base superimpositions yield information of facial displacements relative to the cranial base. The pioneer works of Baumrind et al, ${ }^{2,3}$ Bjork and Skieller, ${ }^{17}$ Ghafari et al, ${ }^{7}$ Halazonetis, 20 and Johnston ${ }^{10}$ showed that displacement of mandibular skeletal and dental components in relation to the maxillary base is critical because the resulting information can differ from conclusions from the cranial superimposition. Future studies are needed to investigate the use of different 3D regional superimposition areas. Currently, superimposition of 3D surface models is still too time-consuming and computing-intensive to apply these methods in routine clinical use. Our current focus is on developing a simplified analysis so that soon these methods can be used clinically.

\section{CONCLUSIONS}

The technique used in this study provides a valid and reproducible 3D assessment of growing patients. The visualization of 3D model superimposition and the surface distance calculations allow a more comprehensive description of treatment outcomes and different patterns of remodeling after treatment.

\section{Acknowledgments}

Supported by NIDCR DE017727 and DE018962.

\section{REFERENCES}

1. Baster M, Rosas A, O'Higgins P. Craniofacial levels and the morphological maturation of the human skull. J Anat 2006;209:637-54. [PubMed: 17062021] 
2. Baumrind S, Korn EL, Isaacson RJ, West EE, Molthen R. Quantitative analysis of the orthodontic and orthopedic effects of maxillary traction. Am J Orthod 1983;84:384-98. [PubMed: 6579840]

3. Baumrind S, Ben-Bassat Y, Bravo LA, Curry S, Korn EL. Partitioning the components of maxillary tooth displacement by the comparison of data from three cephalometric superimpositions. Angle Orthod 1996;66:111-24. [PubMed: 8712489]

4. Buschang PH, Gandini LG Jr. Mandibular skeletal growth and modelling between 10 and 15 years of age. Eur J Orthod 2002;24:69-79. [PubMed: 11887381]

5. Efstratiadis S, Baumrind S, Shofer F, Jacobsson-Hunt U, Laster L, Ghafari J. Evaluation of Class II treatment by cephalometric regional superpositions versus conventional measurements. Am J Orthod Dentofacial Orthop 2005;128:607-18. [PubMed: 16286208]

6. Efstratiadis SS, Cohen G, Ghafari J. Evaluation of differential growth and orthodontic treatment outcome by regional cephalometric superpositions. Angle Orthod 1999;69:225-30. [PubMed: 10371427]

7. Ghafari J, Engel FE. Laster LL.Cephalometric superimposition on the cranial base: a review and a comparison of four methods. Am J Orthod Dentofacial Orthop 1987;91:403-13. [PubMed: 3472459]

8. Ghafari J, Baumrind S, Efstratiadis SS. Misinterpreting growth and treatment outcome from serial cephalographs. Clin Orthod Res 1998;1:102-6. [PubMed: 10321138]

9. Haralabakis NB, Halazonetis DJ, Sifakakis IB. Activator versus cervical headgear: superimpositional cephalometric comparison. Am J Orthod Dentofacial Orthop 2003;123:296-305. [PubMed: 12637902]

10. Johnston LE Jr. Balancing the books on orthodontic treatment: an integrated analysis of change. $\mathrm{Br}$ J Orthod 1996;23:93-102. [PubMed: 8771331]

11. Cevidanes LH, Bailey LJ, Tucker GR Jr, Styner MA, Mol A, Phillips CL, et al. Superimposition of 3D cone-beam CT models of orthognathic surgery patients. Dentomaxillofac Radiol 2005;34:36975. [PubMed: 16227481]

12. Cevidanes LH, Styner MA, Proffit WR. Image analysis and superimposition of 3-dimensional conebeam computed tomography models. Am J Orthod Dentofacial Orthop 2006;129:611-8. [PubMed: 16679201]

13. Cevidanes LH, Franco AA, Gerig G, Proffit WR, Slice DE, Enlow DH, et al. Assessment of mandibular growth and response to orthopedic treatment with 3-dimensional magnetic resonance images. Am J Orthod Dentofacial Orthop 2005;128:16-26. [PubMed: 16027621]

14. Yushkevich PA, Piven J, Hazlett HC, Smith RG, Ho S, Gee JC, et al. User-guided 3D active contour segmentation of anatomical structures: significantly improved efficiency and reliability. Neuroimage 2006;31:1116-28. [PubMed: 16545965]

15. Rueckert D, Sonoda LI, Hayes C, Hill DLG, Leach MO, Hawkes DJ. Nonrigid registration using free-form deformations: application to breast MR images. IEEE Trans Med Imaging 1999;18:71221. [PubMed: 10534053]

16. Ford EHR. Growth of the human cranial base. Am J Orthod 1958;44:498-506.

17. Melsen B. The cranial base. Acta Odontol Scand 1974;32(Suppl 62):86-101.

18. Björk A, Skieller V. Normal and abnormal growth of the mandible. A synthesis of longitudinal cephalomettic implant studies over a period of 25 years. Eur J Orthod 1983;5:1-46. [PubMed: 6572593]

19. Chapuis J, Schramm A, Pappas I, Hallermann W, Schwenzer-Zimmerer K, Langlotz F, et al. A new system for computer-aided preoperative planning and intraoperative navigation during corrective jaw surgery. IEEE Trans Inf Technol Biomed 2007;11:274-87. [PubMed: 17521077]

20. Halazonetis DJ. Computer-assisted cephalometric analysis. Am J Orthod Dentofacial Orthop 1994;105:517-21. [PubMed: 8166104] 


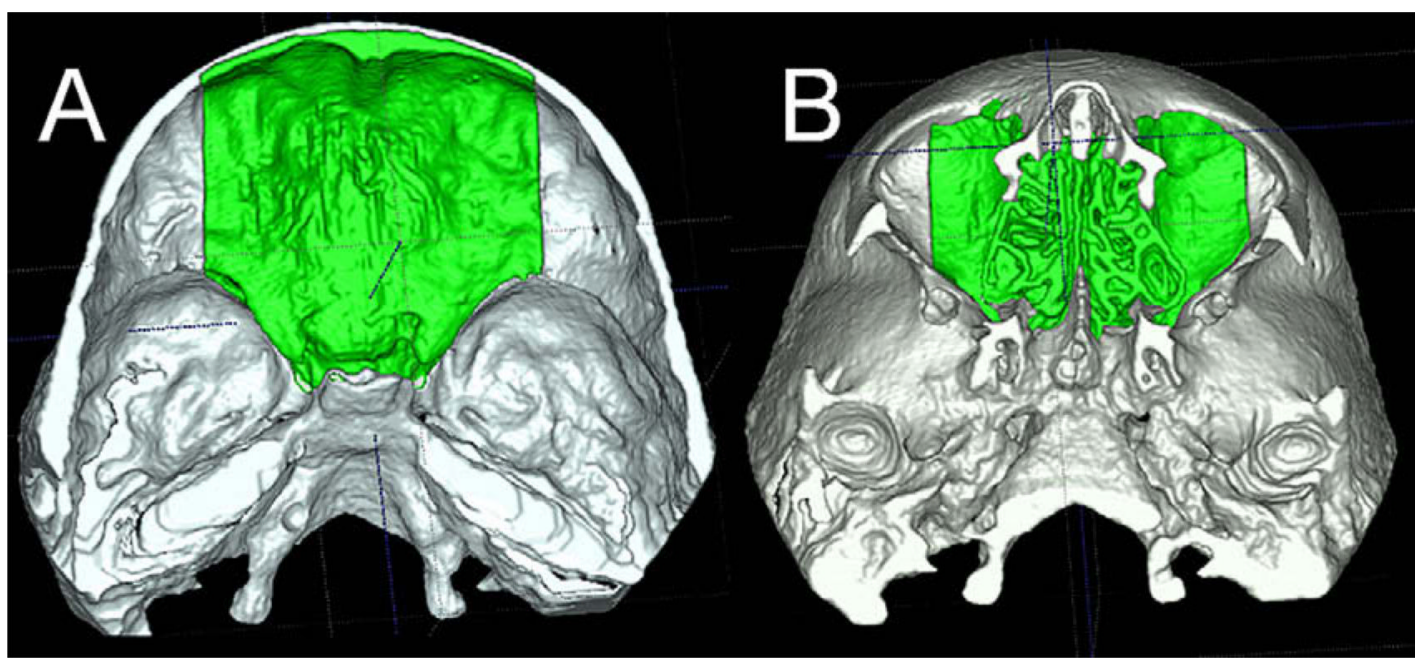

Fig 1.

Anatomic structures of anterior cranial fossa region of the cranial base 3D surface models after treatment that were used for registration: A, superior view; $\mathbf{B}$, inferior view. 


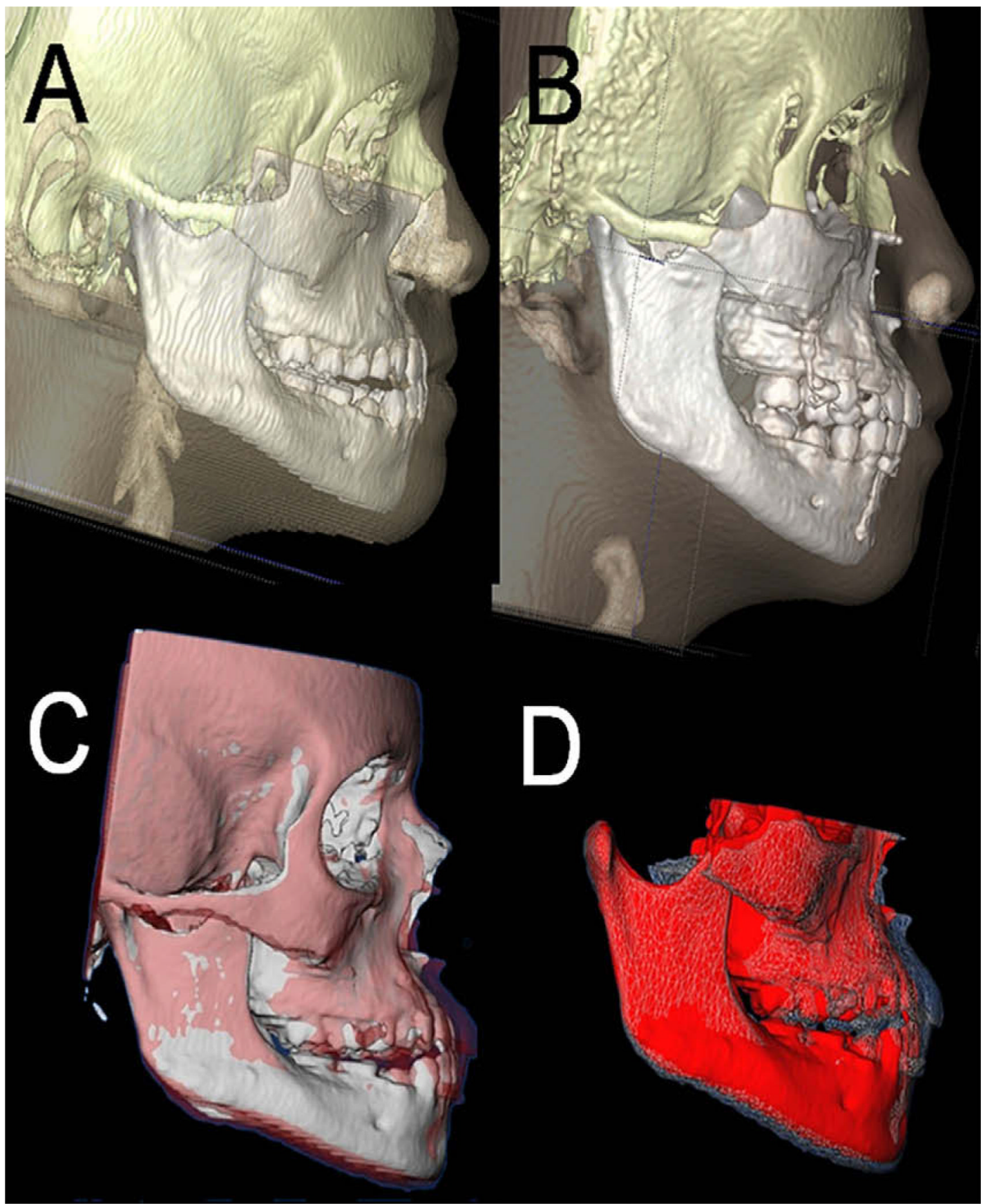

Fig 2.

Overlay of 3D surface models: A and B, pretreatment and posttreatment 3D models; $\mathbf{C}$ and D, registered overlay of 3D models $(C$, pretreatment model [white] and posttreatment [semitransparent red]; $D$, pretreatment $[\mathrm{red}]$ and posttreatment [triangular mesh]). The cranial base was cropped to show details of maxillomandibular changes. 


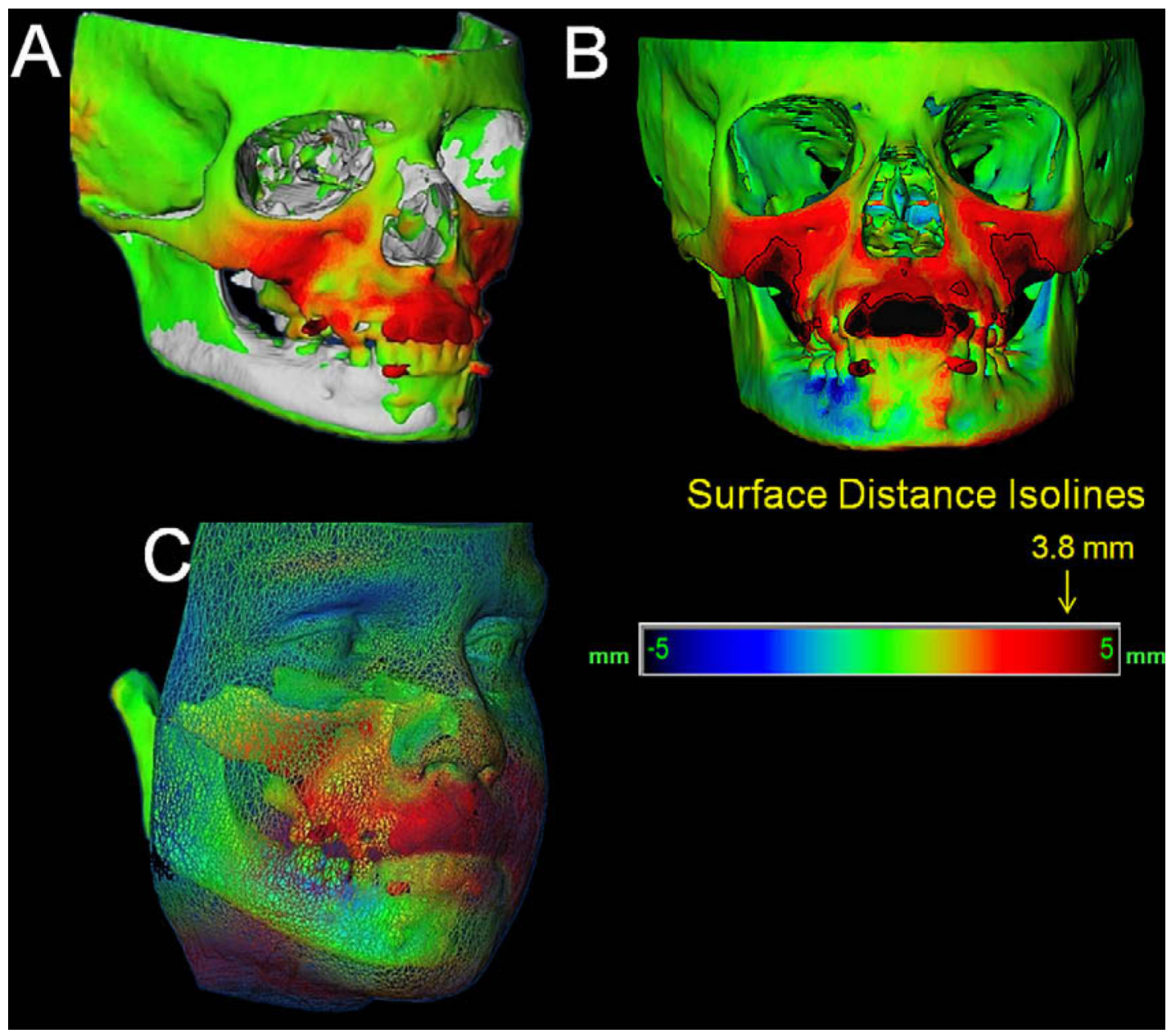

Fig 3.

Quantification of changes: A, pretreatment (white) and posttreatment 3D models (surface distance changes color map). Anterior displacement or remodeling is shown in red, and posterior displacement or remodeling in blue. B, Color maps of hard- and soft-tissue regional changes. $\mathbf{C}$, Isoline contours adjusted to quantify changes in the zygomatic process of the maxilla. 


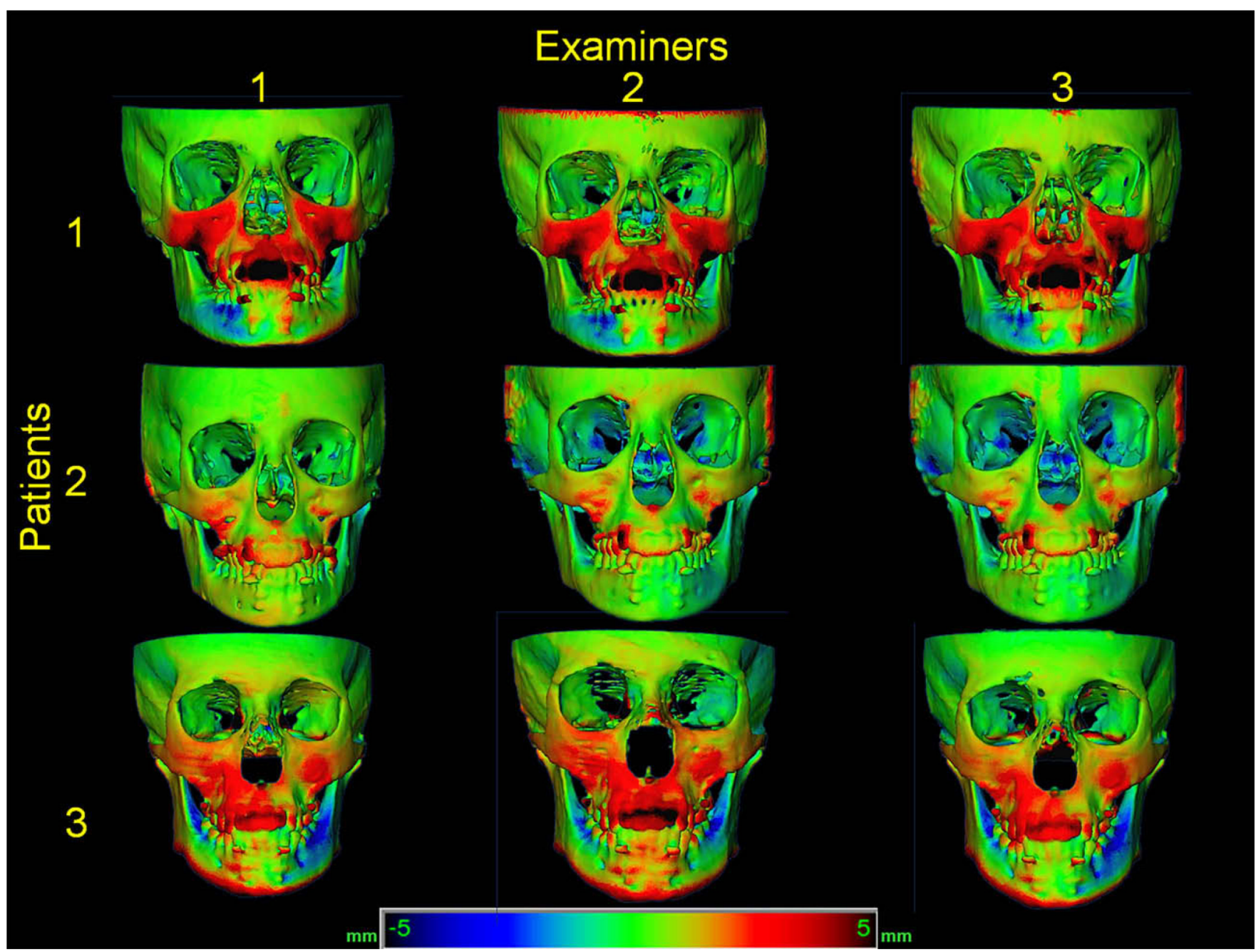

Fig 4.

Skeletal changes in 3D superimpositions of pretreatment and 1-year follow-up of treatment for 3 patients by 3 examiners. 


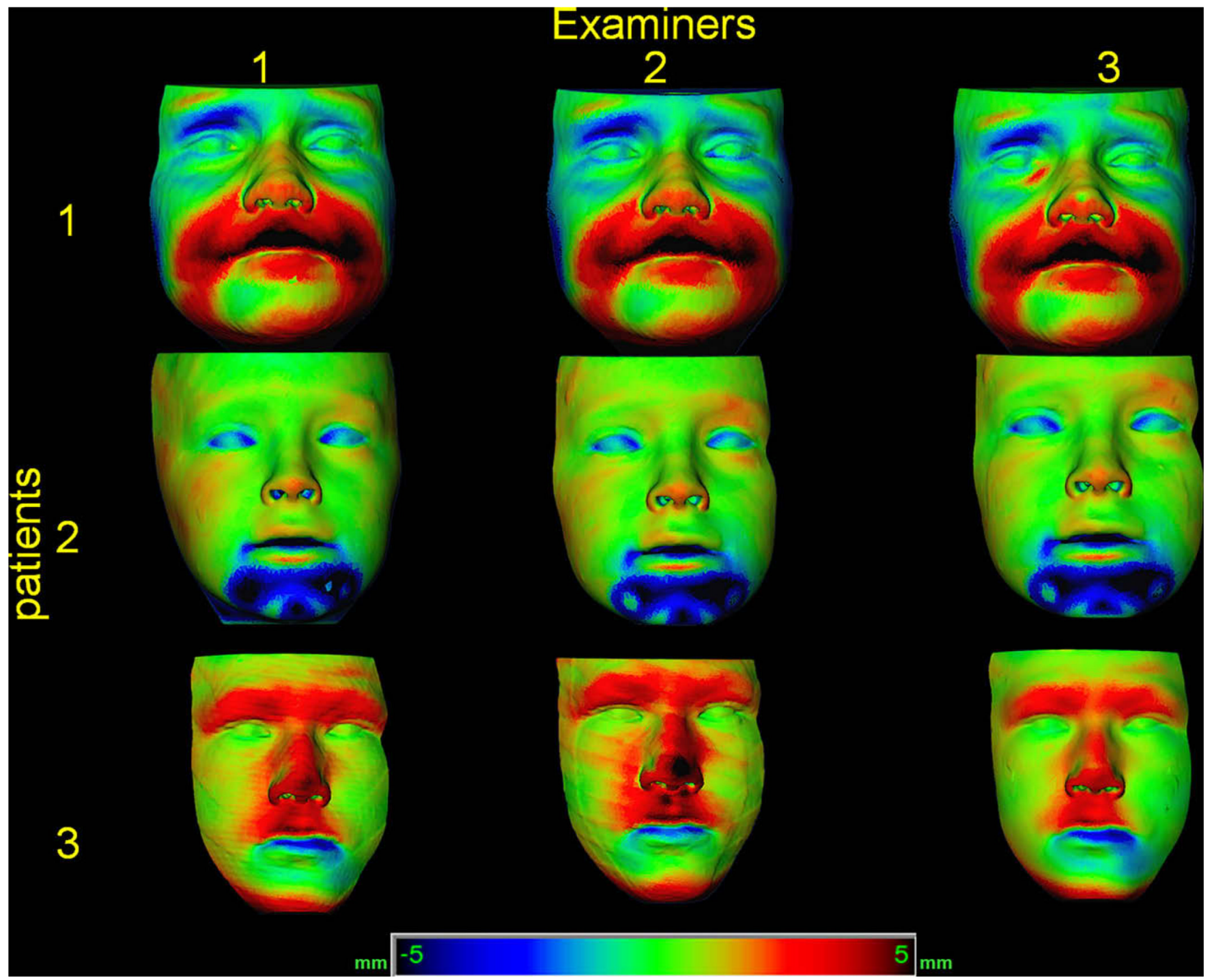

Fig 5.

Soft-tissue changes in 3D superimpositions of pretreatment and 1-year follow-up of treatment for 3 patients by 3 examiners. Patient in the middle row used the chin positioner during the first CBCT scan. 


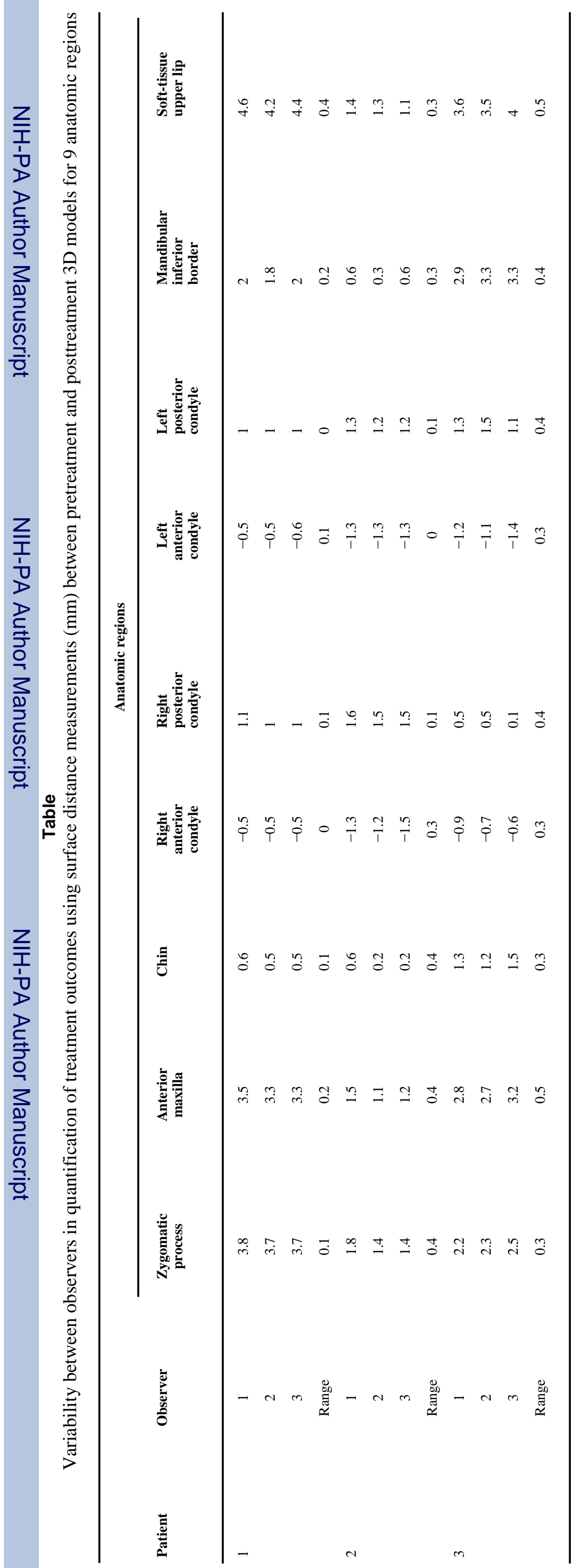

